\title{
Performance Comparisons of Current Metaheuristic Algorithms on Unconstrained Optimization Problems
}

\author{
Umit Can ${ }^{1}$, Bilal Alatas ${ }^{2}$ \\ ${ }^{1}$ Computer Engineering Department, Munzur University, 62000, Tunceli, Turkey, ucan@ munzur.edu.tr \\ ${ }^{2}$ Software Engineering Department, Firat University, 23119, Elazig, Turkey, balatas@ firat.edu.tr
}

\begin{tabular}{l} 
Article Info \\
\hline Article history: \\
Received May $30^{\text {th }}, 2017$ \\
Revised Aug $20^{\text {th }}, 2017$ \\
Accepted Oct $18^{\text {th }}, 2017$ \\
\hline
\end{tabular}

\section{Keyword:}

Metaheuristic Algorithms, Global Optimization, Performance

\begin{abstract}
Nature-inspired metaheuristic algorithms have been recognized as powerful global optimization techniques in the last few decades.Many different metaheuristic optimization algorithms have been presented and successfully applied to different types of problems. In this paper; seven of newest metaheuristic algorithms namely, Ant Lion Optimization, Dragonfly Algorithm, Grey Wolf Optimization, Moth-Flame Optimization, Multi-Verse Optimizer, Sine Cosine Algorithm, and Whale Optimization Algorithm have been tested on unconstrained benchmark optimization problems and their performances have been reported. Some of these algorithms are based on swarm while some are based on biology and mathematics. Performance analysis of these novel search and optimization algorithms satisfying equal conditions on benchmark functions for the first time has given important information about their behaviors on unimodal and multi-modal optimization problems. These algorithms have been recently proposed and many new versions of them may be proposed in future for efficient results in many different types of search and optimization problems.
\end{abstract}

\section{Corresponding Author:}

Umit Can,

Computer Engineering Department,

Munzur University, 62000, Tunceli, Turkey,

ucan@munzur.edu.tr

\section{Introduction}

Optimization is the process of searching for the optimal solution. Analytical, enumeration, and heuristic methods can be used for optimization task. Heuristic refers to experience-based techniques for problemsolving and learning. Heuristics are problem-dependent and designed only for the solution of a specific problem. A metaheuristic is a higher level heuristic that may provide a sufficiently good solution to any optimization problem [1]. Metaheuristic algorithms are simple to implement and faster than the classical calculus based optimization algorithms, they are capable of achieving good approximation to the global optimum, and they are robust to problem changes.

Metaheuristics are recently getting stronger and increasing their popularity due to their advantages. They are used extensively in various fields such as management, computer, engineering due to advantages such as not having difficult mathematical models to set up, good computing powers, and requiring no change on the interested problems like classical algorithms [2]. The different properties of metaheuristic algorithms cause them to perform unequivocally in different optimization problems, and for this reason none of them can be superior to others in all situations. Each has different solutions and superiorities. Their modified new versions 
or novel metaheuristic algorithms are still proposed due to the absence of the most efficient method for all types of problems [3].

There are many metaheuristic optimization methods that are based on biology, physics swarm, sociology, music, chemistry, sports, mathematics and plants. In this paper, seven of newest metaheuristic algorithms namely, Ant Lion Optimization (ALO) [4], Dragonfly Algorithm (DA) [5], Grey Wolf Optimization (GWO) [6], Moth-Flame Optimization (MFO) [7], Multi-Verse Optimizer (MVO) [8], Sine Cosine Algorithm (SCA) [9], and Whale Optimization Algorithm (WOA) [10] have been tested on unconstrained benchmark optimization problems and their performances have been reported.

Organization of this paper has been as follows: Section 2 gives brief explanations of the current algorithms with pseudo-codes. Section 3 gives the descriptions of the unconstrained unimodal and multi-modal benchmark functions. Section 4 reports the experimental results obtained from these metaheuristic algorithms on unconstrained benchmark optimization problems. Section 5 concludes the paper along with future research directions.

\section{Current Metaheuristic Algorithms}

As a constant source of inspiration, nature continues to offer researchers new ideas for new efficient optimization algorithms. In the past decades, various metaheuristic intelligence optimization algorithms have been proposed to solve complex search and optimization problems. These algorithms have shown an outstanding performance on the problems.Hence, while many researchers have focused in adapting them on different problems or to improve their performances, some researchers have proposed novel algorithms inspired from biology, swarm, physics, and etc.

ALO is a nature-inspired algorithm mimicking the hunting behavior of antlions in nature [4].ALO is implemented in five main steps: random walks of ants, building pits, entrapment of ants, catching preys, and lastly rebuilding pits. Pseudo-code of ALO is depicted in Figure 1.

\begin{tabular}{|l|}
\hline Initialize problem parameters \\
Initialize algorithm parameters \\
Initialize the first population of ants and ant lions \\
Determine the best ant lion and assume it as the elite \\
While termination criteria not satisfied \\
For each ant \\
Select and ant lion with roulette wheel method \\
Create a random walk normalize it \\
Update the position of ant \\
End For \\
Compute the fitness of all ants \\
Replace an ant lion with its corresponding fitter ant \\
Update elite if ant lion becomes fitter than the elite \\
End While \\
Return elite
\end{tabular}

Figure 1. Pseudo-code of ALO

DA is inspired from the static and dynamic swarming behaviors of dragonflies in nature [5]. Two essential phases in optimization, namely exploration and exploitation, are designed by modelling the social interaction of dragonflies in navigating, searching for foods, and avoiding enemies when swarming dynamically or statistically [5]. Pseudo-code of DA is shown in Figure 2. 


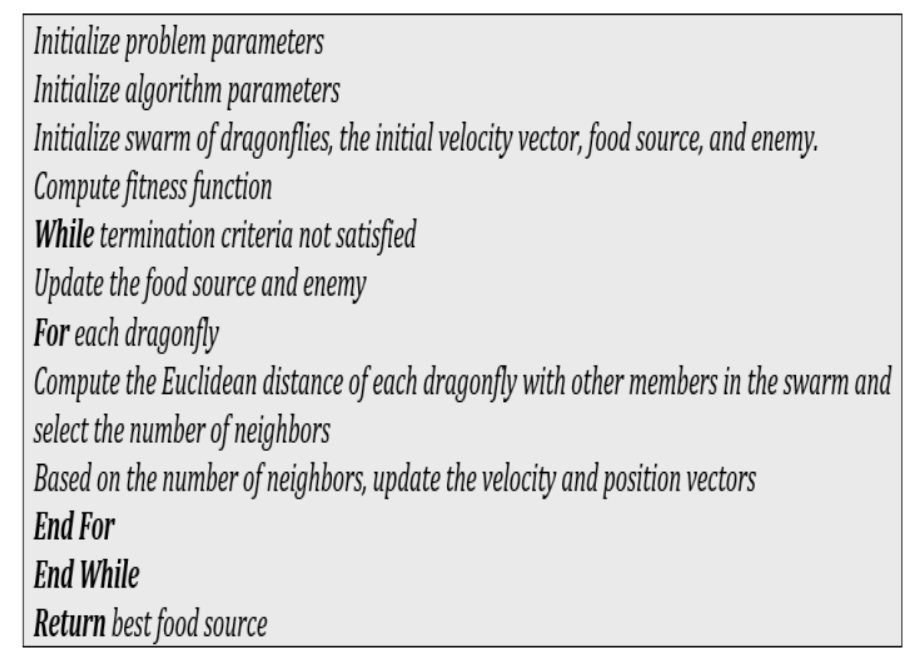

Figure 2. Pseudo-code of DA

GWO mimics the leadership hierarchy and hunting mechanism of gray wolves in nature [6]. GWO has three main steps of hunting, searching for prey, encircling prey, and attacking prey. Pseudo-code of GWO is shown in Figure 3.

MFO is inspired from navigation method of moths in nature called transverse orientation [7]. Spiral flying path of moths around artificial lights (flames) has been mathematically modeled in MFO. Main steps of this algorithm are demonstrated in Figure 4.

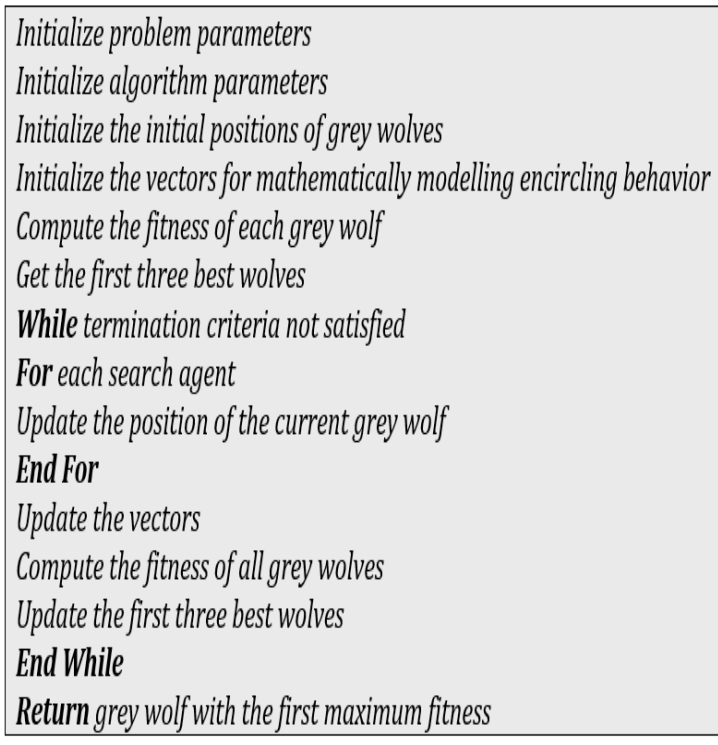

Figure 3. Pseudo-code of GWO 


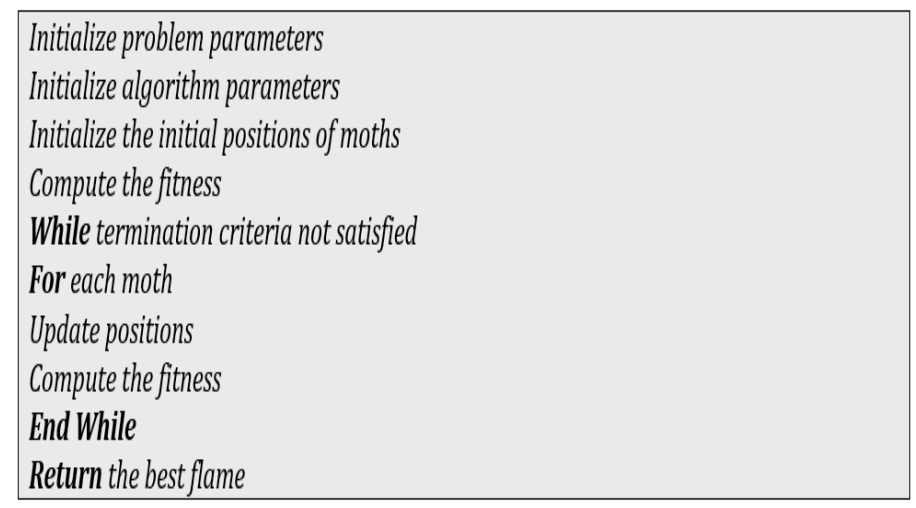

Figure 4. Pseudo-code of MFO

MVO is a novel search and optimization algorithm main inspirations of which are based on three concepts in cosmology: white hole, black hole, and wormhole [8]. The mathematical models of these three concepts are developed to perform exploration, exploitation, and local search, in optimization respectively. Main steps of MVO are demonstrated in Figure 5.

\begin{tabular}{|l|}
\hline Initialize problem parameters \\
Initialize algorithm parameters \\
Initialize galaxy \\
While termination criteria not satisfied \\
Apply Black/White Hole mechanism \\
Apply Wormholes mechanism \\
End While \\
Return best universe position \\
\hline
\end{tabular}

Figure 5. Pseudo-code of MVO

SCA is an interesting math inspired search and optimization algorithm and uses mathematical model based on sine and cosine functions. SCA adaptively balances the exploration and exploitation phases in optimization quickly [9]. Main steps of SCA are demonstrated in Figure 6.

Initialize problem parameters
Initialize algorithm parameters
Initialize search agents
While termination criteria not satisfied
Compute the fitness
Update the next position region between solution and destination, parameters that tells
how far the movement should be towards or outwards the destination, random weight for
destination, and value controlling switches between sine and cosine component.
Update the positions
End While
Return best result

Figure 6. Pseudo-code of SCA

WOA is inspired from the bubble-net hunting strategy [10]. The mathematical model of WOA is based on encircling prey, bubble net hunting, and searching the prey. Main steps of WOA are demonstrated in Figure 7. 


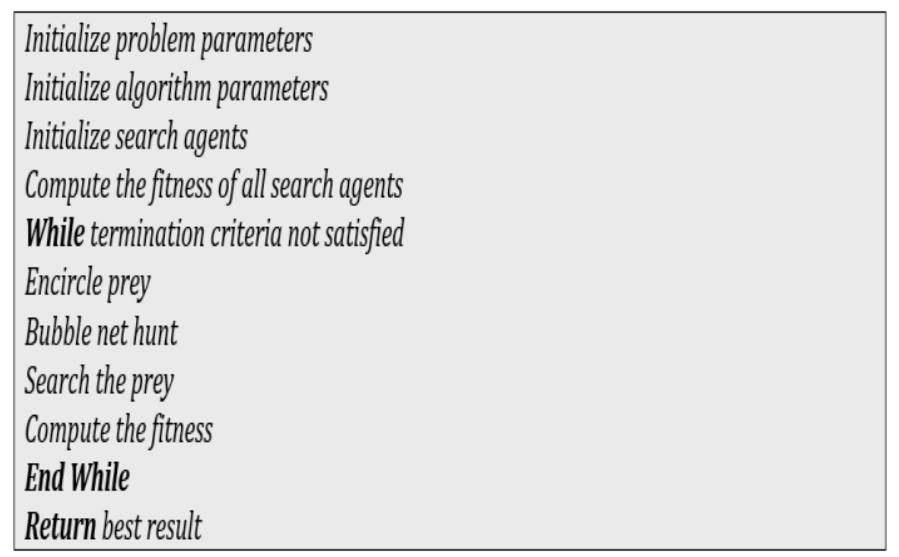

Figure 7. Pseudo-code of WOA

\section{Benchmark Functions}

To evaluate the performance of these seven current metaheuristic algorithm, five benchmark test functions with different characteristics have been used. These benchmark functions are frequently used to evaluate and compare the characteristics of optimization algorithms in terms of convergence, precision, robustness, and general performance. The nature, complexity, and other properties of these benchmark functions can be easily obtained from their definitions and the difficulty levels of these benchmark functions can be adjusted by changing their dimension and interval parameters [11].

Chung Reynolds functionis a unimodal function with less complexity and it can be used to evaluate the converging behaviors of algorithms [12]. Its graph with two dimensions has been shown in Figure 8. Griewank function is a multi-modal function with many local optima and it can be used to test the global search ability of the optimization algorithms in avoiding premature convergence [13]. Its graph with two dimensions has been shown in Figure 9. Rastrigin function is highly multi-modal, but locations of the minima are regularly distributed. Its graph with two dimensions has been shown in Figure 10. Ackley is a multi-modal function with deep local optimizations and the variables are independent of each other [14].Its graph with two dimensions has been shown in Figure 11. Rosenbrock function is unimodal with two dimensions however multi-modal with higher dimensions [15]. Two dimensional Rosenbrock function has been shown in Figure 12. Schwefel's 2.22 is another unimodal function. It is separable, non-differentiable, continuous, and convex function [16].Its graph with two variables has been shown in Figure 13. Schwefel is multimodal, asymmetric and separable test function [17] and its graph with two dimensions has been shown in Figure 14.

The selected benchmark functions and their properties have been demonstrated in Table 1 . The dimensions $(n)$ for all benchmark functions have been determined as 10 . 


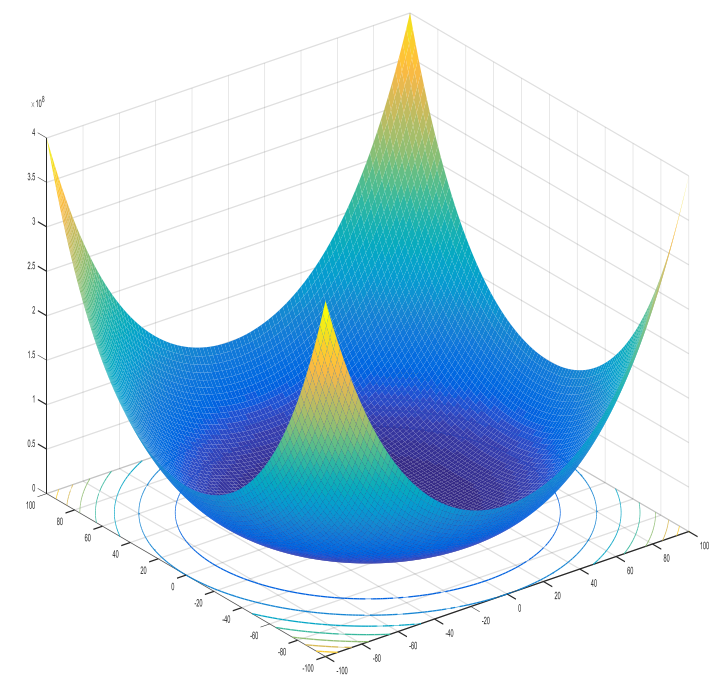

Figure 8. Chung Reynolds function with two variables

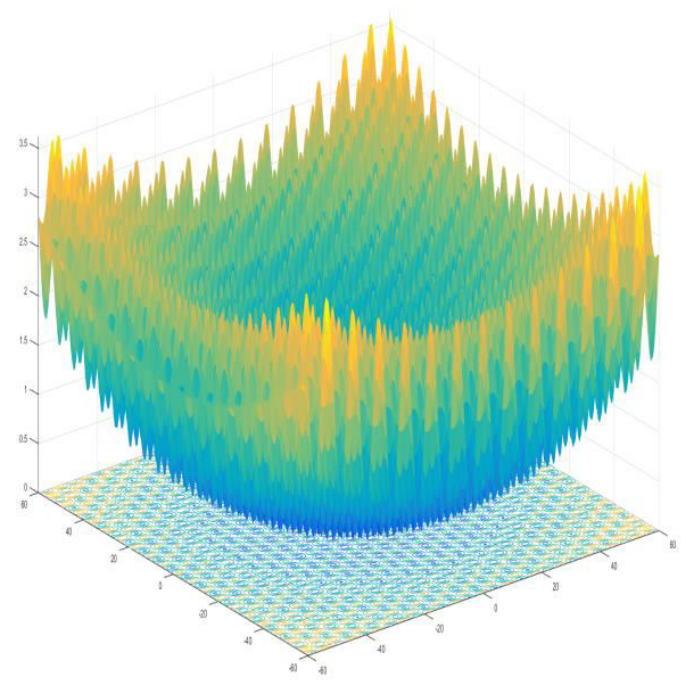

Figure 9. Griewank function with two variables 
Table 1. Benchmark function

\begin{tabular}{|c|c|c|c|}
\hline Function Name & Definition & Interval & $\begin{array}{l}\text { Characteristic } \\
\text { s }\end{array}$ \\
\hline Chung Reynolds & $f_{1}(x)=\left(\sum_{i=1}^{n} x_{i}^{2}\right)^{2}$ & $\begin{array}{l}-100 \leq x_{i} \leq \\
100\end{array}$ & Unimodal \\
\hline Griewank & $f_{2}(x)=1+\frac{1}{4000} \sum_{i=1}^{n} x_{i}^{2}-\prod_{i=1}^{n} \cos \left(\frac{x_{i}}{\sqrt{i}}\right)$ & $\begin{array}{l}-600 \leq x_{i} \leq \\
600\end{array}$ & Multi-modal \\
\hline Rastrigin & $f_{3}(x)=10 \times n+\sum_{i=1}^{n} x_{i}^{2}-10 \cos \left(2 \pi x_{i}\right)$ & $-5 \leq x_{i} \leq 5$ & Multi-modal \\
\hline Ackley & $f_{4}(x)=20+e-20 \cdot e^{-0.2 \cdot \sqrt{\frac{1}{n} \sum_{i=1}^{n} x_{i}^{2}}}-e^{\frac{1}{n} \sum_{i=1}^{n} \cos \left(2 \pi x_{i}\right)}$ & $-32 \leq x_{i} \leq 32$ & Multi-modal \\
\hline Rosenbrock & $\sum_{i=1}^{n-1}\left(100\left(x_{i}^{2}-x_{i+1}\right)^{2}+\left(x_{i}-1\right)^{2}\right)$ & $-30 \leq x_{i} \leq 30$ & Multi-modal \\
\hline Schwefel's 2.22 & $f_{6}(\mathrm{x})=\sum_{i=1}^{n}\left|x_{i}\right|+\prod_{i=1}^{n}\left|x_{i}\right|$ & $\begin{array}{l}-100 \leq x_{i} \leq \\
100\end{array}$ & Unimodal \\
\hline Schwefel & $f_{7}(x)=418.9829 \times n-\sum_{i=1}^{n} x_{i} \sin \left(\left|x_{i}\right|^{\frac{1}{2}}\right)$ & $\begin{array}{l}-500 \leq x_{i} \leq \\
500\end{array}$ & Multi-modal \\
\hline
\end{tabular}

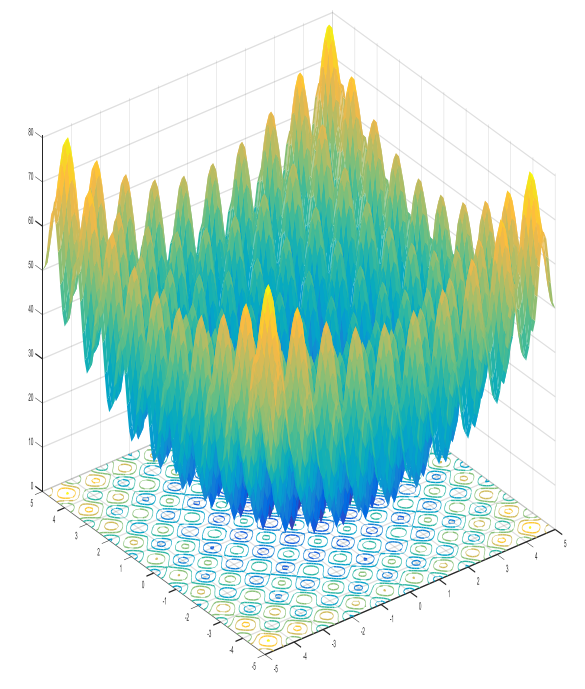

Figure 10. Rastrigin function with two variables 


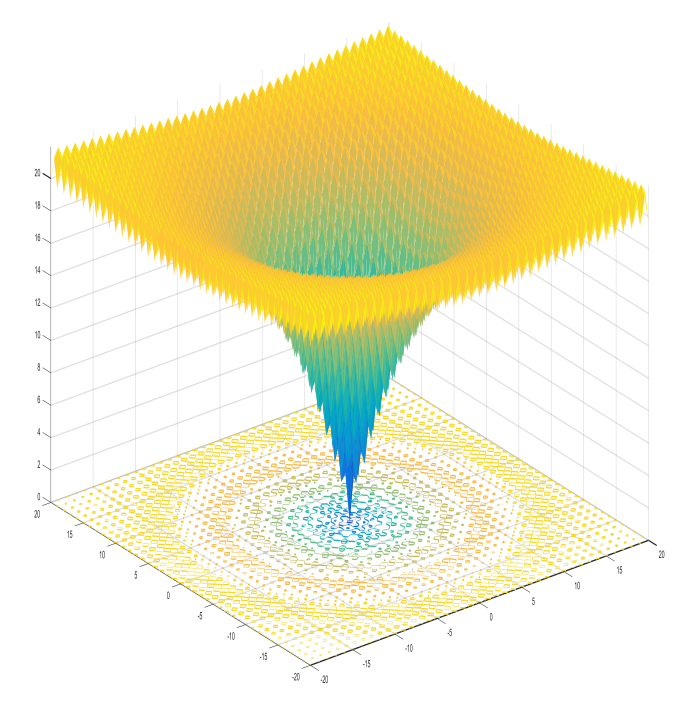

Figure 11. Ackley function with two variables

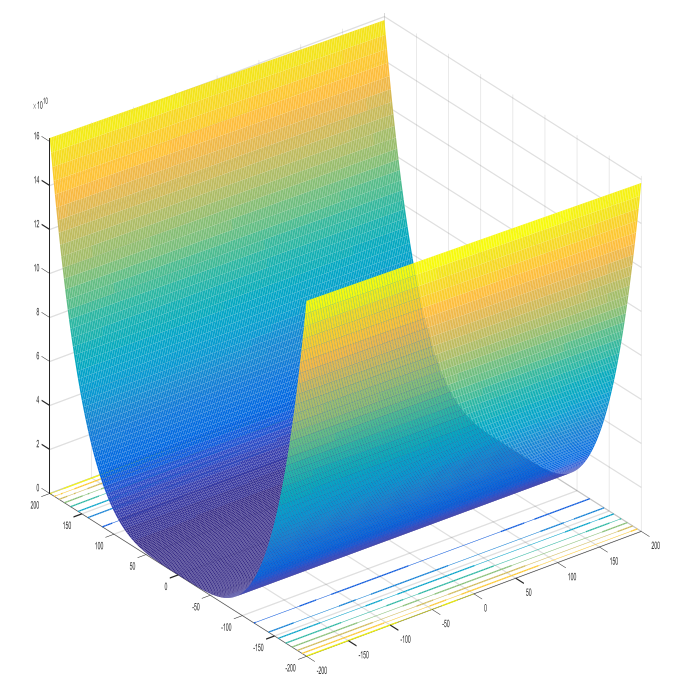

Figure 12. Rosenbrock function with two variables 


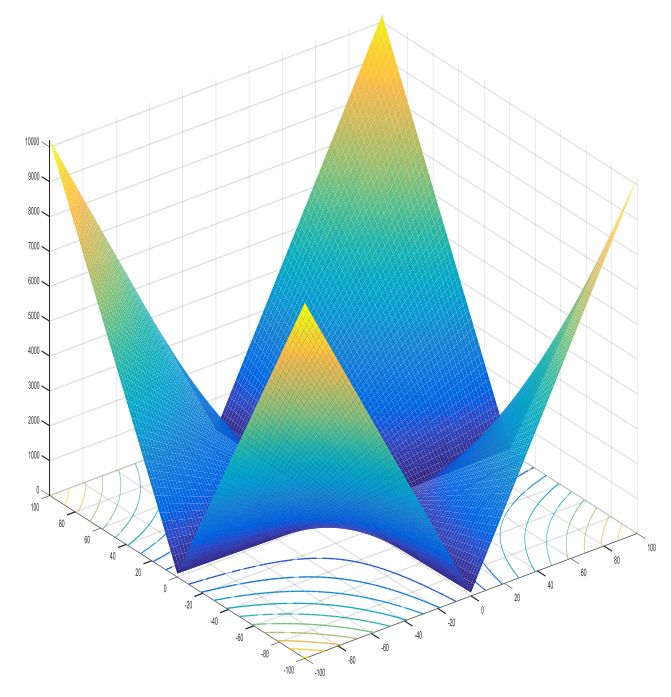

Figure 13. Schwefel's 2.22 function with two variables

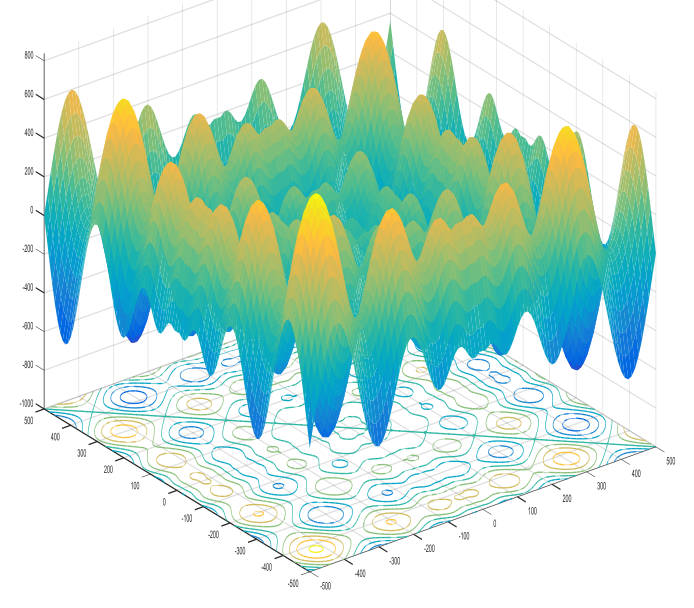

Figure 14. Schwefel function with two variables 


\section{Experimental Results}

The performances of algorithms have been tested on Chung Reynolds function with 10 dimensions. Initial population size of all algorithms is selected as 30 and maximum iteration number for termination criteria is determined as 100. All algorithm parameters have been selected as their original values. All algorithms have been run 10 times. The results obtained from this problem have been demonstrated in Table 2 . From these results, it can be concluded that WOA is the best algorithm within this unimodal problem. GWO is the second best algorithm. MFO seems the worst algorithm within this function using this dimension and interval of the problem and iteration number for the algorithms.

Table 2. Performances of algorithms on Chung Reynolds function

\begin{tabular}{|l|l|}
\hline Algorithm & Mean optimum \\
\hline ALO & 0.3793 \\
\hline DA & 50.2239 \\
\hline GWO & $8.3804 \mathrm{e}-18$ \\
\hline MFO & 88.5679 \\
\hline MVO & 0.0478 \\
\hline SCA & 0.2782 \\
\hline WOA & $2.7393 \mathrm{e}-28$ \\
\hline
\end{tabular}

The performances of algorithms have been tested on Griewank function with 10 dimensions. Initial population size of all algorithms is selected as 30 and maximum iteration number for termination criteria is determined as 100. All algorithm parameters have been selected as their original values. All algorithms have been run 10 times. The results obtained from this problem have been demonstrated in Table 3. From these results, it can be concluded that WOA is the best algorithm within this multi-modal problem. GWO is the second best algorithm.SCA seems the worst algorithm within this function in the selected dimension and interval for the problem and iteration number for the algorithms.

Table 3. Performances of algorithms on Griewank function

\begin{tabular}{|l|l|}
\hline Algorithm & Mean optimum \\
\hline ALO & 0.0913 \\
\hline DA & 0.9221 \\
\hline GWO & $0.0319 \mathrm{e}-4$ \\
\hline MFO & 1.0425 \\
\hline MVO & 0.6848 \\
\hline SCA & 1.0809 \\
\hline WOA & $5.4877 \mathrm{e}-7$ \\
\hline
\end{tabular}

The performances of algorithms have also been tested on another multi-modal problem, namely Rastrigin function, with 10 dimensions. Initial population size of all algorithms is selected as 30 and maximum iteration number for termination criteria is determined as 100. All algorithm parameters have been selected as their original values. All algorithms have been run 10 times. The results obtained from this problem have been demonstrated in Table 4. From these results, it can be concluded that again, WOA is the best algorithm within this multi-modal problem. GWO is the second best algorithm. SCA seems the worst algorithm within this function in the selected dimension and interval for the problem and iteration number for the algorithms. 
Table 4. Performances of algorithms on Rastrigin function

\begin{tabular}{|l|l|}
\hline Algorithm & Mean optimum \\
\hline ALO & 22.9145 \\
\hline DA & 42.7102 \\
\hline GWO & 4.4678 \\
\hline MFO & 15.5878 \\
\hline MVO & 33.9496 \\
\hline SCA & 47.6633 \\
\hline WOA & $1.0747 \mathrm{e}-06$ \\
\hline
\end{tabular}

The performance comparisons of the algorithm on another multi-modal problem, namely Ackley function, with 10 dimensions have been demonstrated in Table 5. Initial population size of all algorithms is selected as 30 and maximum iteration number for termination criteria is determined as 100. All algorithm parameters have been selected as their original values. All algorithms have been run 10 times. From these results, it can be concluded that again, WOA is the best algorithm within this multi-modal problem. GWO is the second best algorithm. DA seems the worst algorithm within this function using the selected dimension and interval for the Ackley function and iteration number for the algorithms.

The performance comparisons of the algorithm on Rosenbrock with 10 dimensions have been demonstrated in Table 6. Initial population size of all algorithms is selected as 30 and maximum iteration number for termination criteria is determined as 100 . All algorithm parameters have been selected as their original values. All algorithms have been run 10 times. From these results, it can be concluded that again, GWO is the best algorithm within this multi-modal problem. WOA is the second best algorithm. DA seems the worst algorithm within this function in the selected dimension and interval for the Rosenbrock function and iteration number for the algorithms.

Table 5. Performances of algorithms on Ackley function

\begin{tabular}{|l|l|}
\hline Algorithm & Mean optimum \\
\hline ALO & 4.1785 \\
\hline DA & 5.6896 \\
\hline GWO & $1.1195 \mathrm{e}-05$ \\
\hline MFO & 3.4870 \\
\hline MVO & 0.3215 \\
\hline SCA & 0.4256 \\
\hline WOA & $1.9207 \mathrm{e}-06$ \\
\hline
\end{tabular}

Table 6. Performances of algorithms on Rosenbrock function

\begin{tabular}{|l|l|}
\hline Algorithm & Mean optimum \\
\hline ALO & 12.7817 \\
\hline DA & 573.5498 \\
\hline GWO & 8.0282 \\
\hline MFO & 299.0972 \\
\hline MVO & 12.7262 \\
\hline SCA & 238.4051 \\
\hline WOA & 8.6148 \\
\hline
\end{tabular}

The performance comparisons of the algorithm on unimodal Schwefel's 2.22 with 10 dimensions have been demonstrated in Table 7. Initial population size of the algorithm is selected as 30 and maximum iteration number for termination criteria is determined as 100. All algorithms have been run 10 times. According to theobtained experimental results, it can be concluded that, WOA is the best algorithm within this unimodal problem. GWO is the second best algorithm. MVO seems the worst algorithm within this function in the selected dimension and interval for the Schwefel's 2.22 function and iteration number for the algorithms. 
Table 7. Performances of algorithms on Schwefel's 2.22 function

\begin{tabular}{|l|l|}
\hline Algorithm & Mean optimum \\
\hline ALO & 332.3281 \\
\hline DA & 210.4979 \\
\hline GWO & $4.5780 \mathrm{e}-05$ \\
\hline MFO & 9.5452 \\
\hline MVO & $1.5024 \mathrm{e}+03$ \\
\hline SCA & 1.4083 \\
\hline WOA & $1.0563 \mathrm{e}-08$ \\
\hline
\end{tabular}

The performance comparisons of the algorithm on multi-modal Schwefel with 10 dimensions have been demonstrated in Table 8. Initial population size of the algorithm is selected as 30 and maximum iteration number for termination criteria is determined as 100. All algorithms have been run 10 times. According to the obtained experimental results, it can be concluded that, MFO is the best algorithm within this multi-modal function. DA is the second best algorithm. SCA seems the worst algorithm within this function in the selected dimension and interval for the Schwefel function and iteration number for the algorithms.

Table 8. Performances of algorithms on Schwefel function

\begin{tabular}{|l|l|}
\hline Algorithm & Mean optimum \\
\hline ALO & $1.2240 \mathrm{e}+03$ \\
\hline DA & 955.3876 \\
\hline GWO & $1.8975 \mathrm{e}+03$ \\
\hline MFO & 792.3943 \\
\hline MVO & $1.2647 \mathrm{e}+03$ \\
\hline SCA & $2.1515 \mathrm{e}+03$ \\
\hline WOA & $1.1430 \mathrm{e}+03$ \\
\hline
\end{tabular}

Table 9 demonstrates the total successes of these algorithms. According to this table, WOA has performed better in five of the seven benchmark functions. GWO has performed better only one and MFO has also performed in only one of the seven functions.

Table 9. General evaluations

\begin{tabular}{|l|l|l|l|}
\hline $\begin{array}{l}\text { Function } \\
\text { Name }\end{array}$ & $\begin{array}{l}\text { Characteri } \\
\text { stics }\end{array}$ & $\begin{array}{l}\text { Best } \\
\text { Algorithm }\end{array}$ & $\begin{array}{l}\text { Worst } \\
\text { Algorithm }\end{array}$ \\
\hline $\begin{array}{l}\text { Chung } \\
\text { Reynolds }\end{array}$ & Unimodal & WOA & MFO \\
\hline Griewank & $\begin{array}{l}\text { Multi- } \\
\text { modal }\end{array}$ & WOA & SCA \\
\hline Rastrigin & $\begin{array}{l}\text { Multi- } \\
\text { modal }\end{array}$ & WOA & SCA \\
\hline Ackley & $\begin{array}{l}\text { Multi- } \\
\text { modal }\end{array}$ & WOA & DA \\
\hline Rosenbrock & $\begin{array}{l}\text { Multi- } \\
\text { modal }\end{array}$ & GWO & DA \\
\hline $\begin{array}{l}\text { Schwefel's } \\
2.22\end{array}$ & Unimodal & WOA & MVO \\
\hline Schwefel & $\begin{array}{l}\text { Multi- } \\
\text { modal }\end{array}$ & MFO & SCA \\
\hline
\end{tabular}




\section{Conclusions}

Novel metaheuristic algorithms are still proposed due to the absence of the most efficient method for all types of search and optimization problems. In this paper, performances of current metaheuristic search and optimization methods have been tested and seven new algorithms, namely Ant Lion Optimization, Dragonfly Algorithm, Grey Wolf Optimization, Moth-Flame Optimization, Multi-Verse Optimizer, Sine Cosine Algorithm, and Whale Optimization Algorithm have been selected for this goal. They have been executed on unconstrained unimodal and multi-modal benchmark optimization problems. According to the obtained results within the benchmark functions, WOA is the best algorithm and GWO is the second best algorithm. WOA has performed better in five of the seven benchmark functions. GWO has performed better in only one and MFO has also performed in only one of the seven functions.

These algorithms are very new computational methods and they can be improved in many ways.More validation studies should be performed to discover the capabilities of these algorithms in dealing with the search and optimization problems. There are positive challenges in terms of efficiency and best possible usage of these algorithms.

\section{References}

[1] Du, Ke-Lin, Swamy, M. N. S. (2016) Search and Optimization by Metaheuristics. Springer.

[2] Akyol, S., Alatas, B. (2012). Güncel Sürü Zekası Optimizasyon Algoritmaları, Nevşehir Bilim ve Teknoloji Dergisi, 1(1), 36-50

[3] Altunbey, F., Alatas, B. (2015). Overlapping community detection in social networks using parliamentary optimization algorithm. International Journal of Computer Networks and Applications, 2(1), 12-19.

[4] Mirjalili, S. (2015). The ant lion optimizer. Advances in Engineering Software. 83. 80-98.

[5] Mirjalili, S. (2016). Dragonfly algorithm: a new meta-heuristic optimization technique for solving singleobjective, discrete, and multi-objective problems. Neural Computing and Applications, 27(4), 1053-1073.

[6] Mirjalili, S., Mirjalili, S. M., \& Lewis, A. (2014). Grey wolf optimizer. Advances in Engineering Software, 69, 46-61.

[7] Mirjalili, S. (2015). Moth-flame optimization algorithm: A novel nature-inspired heuristic paradigm. Knowledge-Based Systems, 89, 228-249.

[8] Mirjalili, S., Mirjalili, S. M., \& Hatamlou, A. (2016). Multi-verse optimizer: a nature-inspired algorithm for global optimization. Neural Computing and Applications, 27(2), 495-513.

[9] Mirjalili, S. (2016). SCA: a sine cosine algorithm for solving optimization problems. Knowledge-Based Systems, 96, 120-133.

[10] Mirjalili, S., Lewis, A. (2016). The whale optimization algorithm. Advances in Engineering Software, 95, 51-67.

[11] Alatas, B. Akin, E. Ozer, A. B (2009) Chaos Embedded Particle Swarm Optimization Algorithms, Chaos, Solitons \& Fractals, vol. 40, pp. 1715-1734.

[12] (2017) C. J. Chung, R. G. Reynolds, "CAEP: An Evolution-Based Tool for Real-Valued Function Optimization Using Cultural Algorithms", International Journal on Artificial Intelligence Tool, 7(3), 239291, 1998

[13] (2017) GEATbx: Examples of Objective Functions. Available: http://www.pg.gda.pl/ mkwies/dyd/geadocu/fcnfun8.html

[14] Ackley, D. (1987) An Empirical Study of Bit Vector Function Optimization. Genetic Algorithms and Simulated Annealing, 170-215.

[15] Shang, Y. W., Qiu, Y. H. (2006). A note on the extended Rosenbrock function. Evolutionary Computation, 14(1), 119-126.

[16] (2017) Ardeh, M. A.Well-known optimization benchmark functions. Available http://benchmarkfens.xyz/benchmarkfens/schwefel222fen.html

[17] (2017) GEATbx: Examples of Objective Functions. Available: http://www.pg.gda.pl/ mkwies/dyd/geadocu/fcnfun7.html 